\title{
Automation in pharmaceutical sector by implementation of artificial intelligence platform: a way forward
}

\author{
Amol Shinde*, Dilip Pawar, Kunal Sonawane
}

Department Medical Affairs and Pharmacovigilance, Unichem Laboratories Ltd., Mumbai, Maharashtra, India

Received: 05 May 2021

Accepted: 29 May 2021

*Correspondence:

Amol Shinde,

Email: amol1.shinde@unichemlabs.com

Copyright: $\odot$ the author(s), publisher and licensee Medip Academy. This is an open-access article distributed under the terms of the Creative Commons Attribution Non-Commercial License, which permits unrestricted non-commercial use, distribution, and reproduction in any medium, provided the original work is properly cited.

\begin{abstract}
Worldwide, there are technological advances that swift automation in several aspects of the pharmaceutical industry such as pharmacovigilance, clinical research, medical affairs, and marketing. Innovative technology like artificial intelligence (AI) emphasizes the massive use of the internet for drug development, drug safety, data analytics, communication marketing, and customer engagement to achieve the goal of pharmaceuticals and patient-centric healthcare. Presently, escalating the number of individual case safety reports (ICSRs) necessitate the support of AI in the transformation of drug safety professional. AI can be transformed and evolve the clinical trial process from the conventional method alongside benefited the cutting cost, enhancing the trial quality, and alleviate trial time by almost half. Today, AI may be efficiently implemented to lower the cost of medical information requests, besides the online chatbots to communicate with health care professionals (HCPs) and consumers. There are numerous forthcoming uses of AI which need to be executed for renovation in the field of pharmaceuticals.
\end{abstract}

Keywords: Artificial intelligence, Clinical research, Pharmacovigilance, Medical affairs, Pharmaceuticals marketing

\section{INTRODUCTION}

Since 1950s, AI has been utilized in sectors like banking and financial industries. Over the past decade, it has significant innovations within many domains like pharmaceutical industries, healthcare, insurance industries. ${ }^{1,2}$ In pharmaceuticals, current challenges like majority of drug requires more than 10-year time to come into market and even clinical trial cost is in billions, at the same time there are often chances it may not succeed in late-stage trials. In pharmacovigilance (PV) overall budget consumed for case processing expenditure is high, hence it disrupts overall adverse events reporting. In healthcare of developing countries going which has high disease burden, poor infrastructure and insufficient in skilled HCPs, there are several challenges in PV. Hence, it is in such setting that AI has extensive role to enhance efficiency and the speed of drug development even produce advanced technology for developing and prolonging patient lives with safety. ${ }^{1,3,4}$ Healthcare practice will be digitalized and regionalized which will have improved connectivity, accessibility, and convenience for future medical

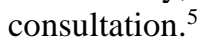

It is essential to recognize innovative technologies that offer the automation of tedious tasks for revolution in pharmaceutical sector. Artificial intelligence (AI) is a subfield of computer science dealing with the simulation of intelligent behaviour in computer and potential of a machine to replicate the performance of human intelligent. It means that the computer programs adroit for solving problems and learning like to human intelligence. ${ }^{6} \mathrm{AI}$ has multiple technologies such as machine learning (ML), natural language processing (NLP) and cognitive services (CI). MI is a technology 'learn' from patterns they identify or ability to understand without openly being programmed and correct behavior appropriately. ${ }^{7}$ It is a broad field in computer science with applications to analyze imaging 
and/or non-imaging patient data and makes assessment of the patient's medical condition, which can be helpful to assist clinicians in their decision-making process. ${ }^{8}$ Natural language processing (NLP) is a technology will analyze, recognize, and produce language that humans use naturally. Cognitive services are a blend of MI and NLP that purpose to resolve certain task that would or else need human intervention. For developing cognitive services, data used to teach the cognitive service, must be prepared, and created. At many decision steps in PV process, cognitive services are identified and developed to support pharmacovigilance users. $^{7}$

Automation of jobs and AI have generated discussion across all industries that are either affected by or considering developing this technology to aid in their extensive functions. One of the mostly discussed questions is whether AI will replace humans in their roles. ${ }^{9}$ One school of thought believes the use of AI will not result in widespread unemployment and humans losing their jobs but will instead create jobs. Research suggests that companies that invest in smart, automated, and selfteaching systems are creating jobs. ${ }^{10}$

\section{METHODS}

The information for this review article were retrieved by using electronic database such as "PubMed", "Google Scholar" and "Google". The articles were searched till January 2020 and the key words used in literature search were limited to AI platform utilizes in clinical research, pharmacovigilance, medical affairs, and marketing of pharmaceutical industry. In this review, we emphasize on automation in several conventional aspects of pharmaceutical industry and improvement in extensive processes of pharmacovigilance, clinical research, medical affairs, and pharmaceuticals marketing.

\section{Use AI in pharmacovigilance}

The World Health Organization defines pharmacovigilance (PV) as "the science and activities relating to the detection, assessment, understanding, and prevention of adverse effects or any other drug-related problem". ${ }^{11}$ Now, drug safety (DS) is a major threat after launching the new drug to the market. During the clinical trial or postmarketing surveillance (PMS), the major source of loss is unpredictable toxicities that cause morbidity and mortality from normal dose of the drug. As per the record from 2008 to 2017, the Food and Drug Administration (FDA) approved 321 novel drugs. At the same time FDA, adverse event (AEs) reporting system has reported a total of more than 10 million AE reports in which 5.8 million recorded as serious, and 1.1 million were death reports. Adverse drug reactions (ADR) data need to be collected by license holder that should be from pharmaceutical industry and submitted to the local drug regulatory authority. The most crucial task in PV is detection and reporting of ADRs, coding of AE in technical terms, preparing individual case safety report (ICSR), assessment of seriousness, and relationship with suspected drug. It is all depend on the human interference, which is time-consuming. Hence, the detection and analysis of ADRs requires a new technology. ${ }^{12}$

Despite in growing cases there is still extensive underreporting of spontaneous AEs. A strategy to defeat underreporting AEs could be social media and health related social networks data sources. There may be adults are using to discuss health related their experiences, such as the use of prescription drugs, side effects, and treatments which makes social network unique source of patient's health information. ${ }^{13}$ Furthermore, that using social digital media does not appear to add anything to what is already known from spontaneous and clinical trial AEs. ${ }^{14}$

As individual case safety report volumes increase, case processing activities constitute a significant portion of internal PV resource use. ${ }^{1,7}$ So, additional expenditures related to contract out are considered, case processing spending, consumes most of a pharmaceutical industry overall PV budget. Automation of AE case processing through AI signifies an opportunity to affect the strongest PV cost driver. ${ }^{15}$ Increases in the number of ICSR require assistive technologies such as AI to support the drug safety (DS) professional with the increasing volume and complexity of work. ${ }^{6}$ AI can be a means to help mitigate complex decision making for PV professionals. $^{7}$ Leveraging AI, the DS professional's work life may potentially change as their decision making is augmented and efficiency enhanced. DS professionals may need to learn new skills and competencies to understand and work with AI. ${ }^{6}$

Electronic health records (EHRs), a more promising external data source which generally provide more complete and thorough representations of patient health that can include clinical narratives such as symptoms, disease status, severity, confounding factors. ${ }^{16}$ Although EHRs are considered a robust source of health information, it is estimated that only $1 \%$ of AEs within EHRs are reported to federal databases. ${ }^{17}$ EHRs represents additional and expanding store of knowledge that could house the presence of impactful AEs. The rapid growth of electronically available health related information, and the ability to process large volumes of them automatically, using natural language processing (NLP) and machine learning algorithms, have opened new opportunities for pharmacovigilance.

AI is beneficial in PV for extraction of accurate safety information. AI tools can automate or facilitate almost every aspect of PV in case processing, risk tracking, which reduces the total processing time and cost. There are some different databases tools, such as VigiFlow, VigiBase, VigiAccess, and VigiLyze. Also, some methods tools i.e. VigiGrade, VigiMatch, and VigiRank for case report analysis. ${ }^{18}$ 
The upcoming approaches of drug safety could become more advanced, driven by AI techniques. Further research is required in the field of AI with respect to PV. The databases, and tools are in primary stage of development, and it could proof its advancement in future in the field of PV.

\section{Use AI in drug design and clinical research}

The new and prominent AI technology is increasing role at various stages of drug development, new drug designing, improving the research and development efficiency, and enhancing the decision-making process to enroll patients for clinical trials. Thus, decreasing the human workload as well as achieving targets in a short time. ${ }^{19}$ Implementation of $\mathrm{AI}$ in the development of a pharmaceutical product from the bench to the bedside can be predicted given that it can aid rational drug design, decision making assistance, establish the appropriate treatment for a patient, including personalized medicines, manage the clinical data produced and utilize it for future drug development. ${ }^{20,21}$ Hence, AI assistance to pharmaceutical industries helps to improve overall life cycle of product.

The enormous chemical space consists of more than 1060 molecules, promotes the development of a larger number of drug molecules. ${ }^{19}$ However, the lack of advanced technologies limits the drug development process, making it a time-consuming and expensive task, which can be addressed by using $\mathrm{AI} .^{22}$ It can acknowledge lead compounds and offer a faster validation of the drug target and optimization of the drug structure design. ${ }^{20,23}$ The traditional process of discovering and developing a drug can take over a decade and costs US $\$ 2.8$ billion on average. Even then, nine out of ten therapeutic molecules do not pass Phase II clinical trials and regulatory approval. ${ }^{24,25}$ Preclinical discovery of molecules and predicting lead compounds before the initiate the clinical trials by implementation of AI, such as predictive ML and other reasoning techniques, that assist in the prompt prediction of lead molecules that would pass clinical trials with consideration of the patient population. ${ }^{26}$

Clinical trials are focused for determining the safety and efficacy of a drug product in humans for a certain indication and require 6 to 7 years along with a huge financial investment. Though, only one out of ten molecules come into these trials, gain successful clearance, which is a massive loss for the pharmaceuticals industry. ${ }^{27}$ These breakdowns can result from inappropriate patient selection, scarcity of technical requirements, and poor infrastructure. However, with the massive digital medical data available, these disappointments can be decreased with the application of AI. ${ }^{26}$ The patients enrolment requires one-third of the clinical trial timeline. The accomplishment of a clinical trial can be ensured by the enrolment of appropriate patients, which otherwise leads to approx. 86 percent of failure cases. ${ }^{28}$
AI technology can support in selecting only a specific diseased population for enrolment in phase II and III of clinical trials by using patient specific genome-exposome profile analysis, which can help out in early prediction of the available drug targets in the patients selected. ${ }^{20,26}$ Drop out of patients from clinical trials accounts for the failure of 30 percent of the clinical trials, producing additional enrolling requirements for the completion of the trial, leading to a wastage of time and money. This can be avoided by close monitoring of the patients and helping them follow the desired protocol of the clinical trial. ${ }^{28}$ Mobile software was developed by AiCure that monitored regular medicine intake by patients with schizophrenia in a phase II trial, which increased the adherence rate of patients by $25 \%$, ensuring successful completion of the clinical trial. ${ }^{20}$

Clinical necessity and availability of huge data have demanded a focus on virtualization and adoption of $\mathrm{AI}$ technology. These advanced technologies are diverse and though most have not been studied in randomized trials, their incorporation across all types of clinical trials is becoming more common place with increasing trial complexity. ${ }^{29,30,31}$ Diversity and evolution in AI technology have preceded to a wide range of potential applications in clinical research. Natural language processing (NLP), the ability of programmed computers to analyze written and spoken word, in addition to machine learning, which looks at algorithms that assist in predictive decision making without explicit programming, can accelerate the patient enrolment process by recognizing appropriate patients through analysis of electronic medical records. ${ }^{26,32,33}$ Application of electronic health records and databases, modeling through NLP and machine learning can potentially identify new biomarkers worth assessing in a more formal context. ${ }^{26}$

In clinical trial conduct where digitalization is being implemented include consent processes, patient adherence, and scheduling through wearable monitoring, medication logging, and virtualization of staff-patient interactions including consultations, study updates, and patient coaching to minimize attrition. ${ }^{34}$

\section{AI use in medical affairs and marketing of pharma- ceutical industry}

In medical affairs, scientific exchange is a main function, hence, medical affairs has always been at the frontline, leading external medical interactions with healthcare professionals (HCPs) as well as other key customers. With science becoming more and more complex, HCPs are looking to pharmaceutical industry for high-level nonpromotional scientific content and engagement. HCPs need to have quick access to scientific content as well as the ability to easily recall it for the future. So, the medical affairs stepped up with the introduction of digital solutions to meet these needs and enrich customer experience. The COVID-19 pandemic has put a focus on the vital role of 
medical information play within a pharmaceutical industry at a time of crisis. ${ }^{35}$

As medical affairs embraces digital transformation, it is important to gain from other industries that have already launched large-scale omnichannel transformations, for example, banking, where the extensive implementation of digital service channels and telecommunications has resulted in $44 \%$ of all customer requests now being handled through digital channels. ${ }^{36}$

An innovative and user-friendly approach to measure the HCP experience, which is a very simple assessment of whether the interaction enables the HCP to get the job done. ${ }^{37}$ The key to satisfying HCP is not just to measure what happens but also to use the data to drive action throughout the industry. ${ }^{38}$

Generally, the consumer does Google search for medical information which may be inaccurate and dangerous. So, we need to ensure we are the first place they go to, and this requires advocating for better regulatory flexibility regarding the digital exchange of medical information. We must figure out a way to tear down the walls between medical and commercial team of pharmaceutical industry and incorporate in a way that works for the customer. When external customers such as, key opinion leaders (KOL), HCPs and consumers contact a pharmaceutical industry looking for information and speedy response. ${ }^{35}$

In pharmaceutical industry, market positioning is the process of establishing a uniqueness of the product in the market to attract consumers to buy them, making it an essential element in almost all business strategies for industries to establish their own unique identity. ${ }^{39,40}$ For example, in the marketing of pioneer brand Viagra, where the market targeted it not only for the treatment of men's erectile dysfunction, but also for other problems affecting quality of life. ${ }^{41}$ With the assistance of advanced technology and e-commerce, it has become easier for pharma industry to get a natural recognition of their brand in the market. Companies develop search engines as one of the innovative platforms to occupy a prominent position in online marketing and help in the positioning of the product in the market, as also confirmed by the internet advertising bureau. Companies continuously try to rank their websites higher than those of other companies, giving recognition to their brand in a short time. ${ }^{42}$

The success of a pharmaceutical industry is in the constant development and business growth. Even with access to huge funds, R\&D output in the pharmaceutical industry is collapsing because of the failure of companies to implement new marketing technologies. ${ }^{43}$ The developments in digital technologies, it is referred to as 'fourth industrial revolution', is helping innovation in digital marketing via a multicriteria decision-making approach, which collects and analyzes statistical and mathematical data and implements human inferences to make AI-based decision-making models discover new marketing methodology. ${ }^{44}$ Prediction of the market is important for various pharmaceutical distribution industries, which can implement AI application in the field, such as 'business intelligent smart sales prediction analysis', which uses a combination of time series forecasting and real-time application. It helps pharmaceutical organization to forecast the sale of products in advance to prevent costs of excess stock or prevent customer loss because of shortages. ${ }^{45}$

\section{DISCUSSION}

In the past few years, there has been tremendous increase in data digitalization in the pharmaceutical industry. However, this digitalization comes with the challenge of acquiring, scrutinizing, and applying that knowledge to resolve complex clinical problems. ${ }^{46}$ It encourages the use of AI platform, because it can manage large volumes of data with enhanced automation. ${ }^{47}$

AI technology encompassing several advanced tools and networks that can mimic human intelligence. At the same time, it does not threaten to replace human intervention completely. ${ }^{48,49}$ AI technology develops systems and software that can interpret and learn from the input data to make independent decisions for accomplishing specific objectives. Its applications are continuously being extended in the pharmaceutical field, as described in this review. According to the McKinsey Global Institute, the rapid advances in AI-guided automation will be likely to completely change the work culture of industry. ${ }^{50,51}$

The entire success of AI depends on the availability of a large amount of data because these data are used for the subsequent training provided to the system. Access to data from various database providers can incur extra costs to a pharmaceutical industry, and the data should also be reliable and high quality to ensure accurate result prediction. Other challenges that prevent full-fledged adoption of $\mathrm{AI}$ in the pharmaceutical industry include the lack of skilled personnel to operate AI technology, limited budget for small organizations, uneasiness of replacing humans leading to job loss, uncertainty about the data produced by AI, and the black box phenomenon that means how the conclusions are reached by the AI technology. ${ }^{51}$

Automation of several tasks in drug development, clinical trials, and sales will take place with time, but these all come under the category of 'narrow AI'; where AI must be trained using a large volume of data and, thus, makes it suitable for a particular task. Therefore, human intervention is required for the successful implementation, development, and operation of the AI platform. However, the fear of unemployment could be a myth given that $\mathrm{AI}$ is currently taking over repetitive jobs, while leaving scope for human intelligence to be used for developing more complicated insights and creativity. Nevertheless, AI technology has been implemented by several pharmaceutical industry, and it is expected that a revenue 
of US\$2.199 billion will be created by 2022 through AIbased solutions in the pharmaceutical sector, with an investment exceeding US\$7.20 billion across 300 plus deals between 2013 and 2018 by the pharmaceutical industry. ${ }^{52}$

In the context of a public health emergency, these AI technology functions could enhance research conduct efficiency but may pose their own ethical challenges especially if applied to non-pandemic circumstances, or if insufficient attention is paid to data security or ethical considerations. ${ }^{53}$

Pharmaceutical industry need clarity about the potential of AI technology in finding solutions to problems once it has been implemented, along with understanding the realistic goals that can be achieved. Skilled data scientists, software engineers with a sound knowledge of AI technology, and a clear understanding of the pharmaceutical business target and its R\&D objective can be developed to use all aspect of AI technology.

\section{CONCLUSION}

The advancement of AI technology, along with its remarkable tools, continuously decrease challenges faced by pharmaceutical industry, impacting the drug development process along with the overall product lifecycle. With the help of AI-based technologies will not only speed up the time needed for the products to come into the market but will also improve the product quality and safety of the production process, provide every aspect of pharmacovigilance in case processing, risk tracking, which reduces the total processing time and cost, and help to mitigate complex decision making for pharmacovigilance, thereby, increasing the importance of automation.

The most important fear regarding the incorporation of these technologies is the job losses that would follow, and the stringent regulations needed for the implementation of AI platform to several important aspect of pharmaceutical industry. Though, these advanced technologies are intended only to make work easier and accurate and not to completely replace humans. In near future, AI technology will become a valuable tool in the pharmaceutical industry.

Funding: No funding sources Conflict of interest: None declared

Ethical approval: Not required

\section{REFERENCES}

1. Schmider J, Kumar K, LaForest C, Swankoski B, Naim K, Caubel P. Innovation in Pharmacovigilance: Use of Artificial Intelligence in Adverse Event Case Processing. Clin Pharmacol Therap. 2018;105(4):95461.
2. Cabitza F, Rasoini R, Gensini G. Unintended Consequences of Machine Learning in Medicine. JAMA. 2017;318(6):517.

3. Nayak V, Khan M, Shukla B, Chaturvedi P. Artificial intelligence in clinical research. Int $\mathrm{J}$ Clin Trials. 2016;3(4):187-93.

4. Hoodbhoy Z, Hasan B, Siddiqui K. Does artificial intelligence have any role in healthcare in low resource settings? J Med Art Intel. 2019;2:13-13.

5. Healthcare in 2065. 2021. Available at: https://www2.deloitte.com/content/dam/Deloitte/sg/D ocuments/risk/sg-risk-healthcare-2065-noexp.pdf. Accessed on 24 January 2021.

6. Danysz K, Cicirello S, Mingle E, Assuncao B, Tetarenko N, Mockute R, et al. Artificial Intelligence and the Future of the Drug Safety Professional. Drug Safety. 2018;42(4):491-7.

7. Mockute R, Desai S, Perera S, Assuncao B, Danysz K, Tetarenko N, et al. Artificial Intelligence Within Pharmacovigilance: A Means to Identify Cognitive Services and the Framework for Their Validation. Pharm Med. 2019;33(2):109-20.

8. Chan H, Hadjiiski L, Samala R. Computer-aided diagnosis in the era of deep learning. Med Physics. 2020;47(5).

9. Frey C, Osborne M. The future of employment: How susceptible are jobs to computerization? 2021. Available at: https://www.oxfordmartin.ox.ac. uk/publications/the-future-of-employment/. Accessed on 24 January 2021.

10. Marr B. Instead of Destroying Jobs Artificial Intelligence (AI) Is Creating New Jobs In 4 Out Of 5 Companies. Forbes. 2021. Available at: https://www.forbes.com/sites/bernardmarr/2017/10/1 2/instead-of-destroying-jobs-artificial-intelligence-aiis-creating-new-jobs-in-4-out-of-5-companies/. Accessed on 24 January 2021.

11. WHO Policy Perspectives on Medicines. Looking at the Pharmacovigilance: ensuring the safe use of medicines. Geneva: World Health Organization. Available at: http://apps.who.int/medic inedo cs/pdf/s6164 e/s6164 e.pdf. Accessed on 15 December 2020.

12. Basile A, Yahi A, Tatonetti N. Artificial Intelligence for Drug Toxicity and Safety. Trends Pharmacol Sci. 2019;40(9):624-35.

13. Sarker A, Ginn R, Nikfarjam A, O’Connor K, Smith $\mathrm{K}$, Jayaraman $\mathrm{S}$, et al. Utilizing social media data for pharmacovigilance: A review. 2015;54:202-12.

14. Caster O, Dietrich J, Kürzinger M, Lerch M, Maskell $\mathrm{S}$, Norén $\mathrm{G}$ et al. Assessment of the Utility of Social Media for Broad-Ranging Statistical Signal Detection in Pharmacovigilance: Results from the WEB-RADR Project. Drug Safety. 2018;41(12):1355-69.

15. Yang C, Yang H, Jiang L. Post marketing Drug Safety Surveillance Using Publicly Available HealthConsumer-Contributed Content in Social Media. 2014;5(1):1-21.

16. Wang X, Hripcsak G, Markatou M, Friedman C. Active Computerized Pharmacovigilance Using 
Natural Language Processing, Statistics, and Electronic Health Records: A Feasibility Study. J Am Med Informat Assoc. 2009;16(3):328-37.

17. Luo Y, Thompson W, Herr T, Zeng Z, Berendsen M, Jonnalagadda $S$ et al. Natural Language Processing for EHR-Based Pharmacovigilance: A Structured Review. Drug Safety. 2017;40(11):1075-89.

18. UMC WHO. Available at: https://www.whoumc.org/about-us/who-we-are/. Accessed on 11 October 2018.

19. Mak KK, Pichika MR. Artificial intelligence in drug development: Present status and future prospects. Drug Discov Today. 2019;24:773-80.

20. Duch W, Swaminathan K, Meller J. Artificial intelligence approaches for rational drug design and discovery. Curr Pharm Des. 2007;13(14):1497-508.

21. Blasiak A, Khong J, Kee T. CURATE.AI: Optimizing Personalized Medicine with Artificial Intelligence. SLAS Technol. 2020;25(2):95-105.

22. Mishra V. Artificial intelligence: the beginning of a new era in pharmacy profession. Asian $\mathrm{J}$ Pharm. 2018;12(2).

23. Sellwood MA. Artificial intelligence in drug discovery. Future Sci. 2018; 2025-8.

24. Álvarez-Machancoses Ó, Fernández-Martínez JL. Using artificial intelligence methods to speed up drug discovery. Expert Opin Drug Disc. 2019;14:769-77.

25. Fleming N. How artificial intelligence is changing drug discovery. Nature. 2018;557:55.

26. Harrer S. Artificial intelligence for clinical trial design. Trends Pharmacol Sci. 2019;40:577-91.

27. Hay M. Clinical development success rates for investigational drugs. Nature Biotechnol. 2014;32:4051.

28. Fogel DB. Factors associated with clinical trials that fail and opportunities for improving the likelihood of success: a review. Contemp Clin Trials Communic. 2018;11:156-64

29. Angus Derek C. Randomized Clinical Trials of Artificial Intelligence. JAMA. 2020;323(11):1043.

30. Parikh RB, Teeple S, Navathe AS. Addressing bias in artificial intelligence in health care. JAMA. 2019;322:2377.

31. Emanuel EJ, Wachter RM. Artificial intelligence in health care: will the value match the hype? JAMA. 2019;321:2281-2.

32. Nayak VS, Khan MS, Shukla BK, Chaturvedi PR. Artificial intelligence in clinical research. Int J Clin Trials. 2016;3(4):187-93.

33. Woo M. An AI boost for clinical trials. Nature. 2019;573(7775):100-2.

34. Taylor K, Properzi F, Cruz MJ Intelligent clinical trials. Deloitte. 2020. Available at: https://www2.deloitte.com/us/en/insights/industry/lif e-sciences/artificial-intelligence-in-clinicaltrials.html. Accessed on 15 April 2020.

35. From Aspiration to Action: Embracing a CustomerCentric Mindset in Medical Affairs Through Digital Transformation. Available at: http://engage.
diaglobal.org/Indegene-Whitepaper-2020.html.

Available at: 25 January 2021.

36. Hedwig M, Friesdorf M, Goryunov Y, Niedermann F. Omnichannel consumer interactions - a payer perspective. Available at: https://www.mckinsey. com/industries/ health care-systems-and-services/ourinsights/omnichannel-consumer-interactions-a-payerperspective. Accessed on 20 May 2020.

37. An Experience Insights Consultancy. Available at: https://gostonemantel.com/. 2020. Accessed on 20 May 2020.

38. Duncan E, Fanderl H, Maechler N, Neher K. Creating value through transforming customer journeys. Winter 2016. Available at: https://www.mckinsey. com/business-functions/marketing-and-sales/ourinsights/customer-experience-creating-value-throughtransforming-customer-journeys. Accessed on 20 May 2020.

39. Kalafatis SP, Tsogas MH, Blankson C. (2000) Positioning strategies in business markets. J Bus Industr Market. 2000;15(6).

40. Jalkala AM, Keränen J. Brand positioning strategies for industrial firms providing customer solutions. J Bus Industr Market. 2014;29(3).

41. Ding M, Eliashberg J, Stremersch S. Innovation and Marketing in the Pharmaceutical Industry, Springer Nature. 2014th edition. 2016.

42. Dou W. Brand positioning strategy using search engine marketing. Mis Quarterly. 2010;261-79.

43. Toker D. A decision model for pharmaceutical marketing and a case study in Turkey. Ekonomska Istraživanja 2013;26:101-14.

44. Singh J. Sales profession and professionals in the age of digitization and artificial intelligence technologies: concepts, priorities, and questions. J Personal Selling Sales Management 2019;39:2-22.

45. Mahajan KN, Kumar A. Business intelligent smart sales prediction analysis for pharmaceutical distribution and proposed generic model. Int J Comp Sci Informat Technol. 2017;8:407-12.

46. Ramesh A. Artificial intelligence in medicine. Ann Royal Coll Surg Engl. 2004;86, 334-8.

47. Miles J, Walker A. The potential application of artificial intelligence in transport. In IEE ProceedingsIntelligent Transport Systems 2006;153:183-98.

48. Yang Y, Siau K. A Qualitative Research on Marketing and Sales in the Artificial Intelligence Age, MWAIS. 2018.

49. Wirtz BW. Artificial intelligence and the public sector-applications and challenges. Int $\mathbf{J}$ Public Admin. 2019;42:596-615.

50. Smith RG, Farquhar A. The road ahead for knowledge management: an AI perspective. AI Magazine. 2000;21:17.

51. Lamberti MJ. A study on the application and use of artificial intelligence to support drug development. Clin Therap. 2019;41:1414-26.

52. Research and markets. Global Growth Insight - Role of AI in the Pharmaceutical Industry 2018-2022: Exploring Key Investment Trends, Companies-to- 
Action, and Growth Opportunities for AI in the Pharmaceutical Industry, Research and Markets. 2019.

53. Madariaga A, Kasherman L, Karakasis K, Degendorfer P, Heesters A, Xu W, et al. Optimizing clinical research procedures in public health emergencies. Med Res Rev. 2020.
Cite this article as: Shinde A, Pawar D, Sonawane $\mathrm{K}$. Automation in pharmaceutical sector by implementation of artificial intelligence platform: a way forward. Int $\mathbf{J}$ Basic Clin Pharmacol 2021;10:863-9. 